\title{
Osteomalácia Oncogênica: Cintilografia com Sestamibi-99mTc na Localização do Tumor Periférico
}

\begin{abstract}
RESUMO
A osteomalácia oncogênica hipofosfatêmica $(\mathrm{OOH})$ é uma síndrome paraneoplásica induzida por tumor, de tecidos mole ou ósseo. Apresenta-se com dor e fraturas, acompanhada de hipofosfatemia, hiperfosfatúria e concentrações plasmáticas de $1,25(\mathrm{OH})_{2} \mathrm{D}_{3}$ inapropriadamente normais/diminuídas. Após a remoção do tumor, a completa resolução das anormalidades clínicas e bioquímicas é sua maior característica. Uma mulher de 44 anos de idade é descrita no caso com dificuldade para caminhar por causa de dores nos membros inferiores, fraqueza muscular generalizada e hipofosfatemia com relativa hiperfosfatúria. A cintilografia de corpo total com sestamibi- ${ }^{99 m} T c$ mostrou acúmulo do radiofármaco no terço superior de coxa esquerda onde pequeno tumor foi detectado no exame pelo ultra-som. Com a retirada do tumor, um lipoma, os sintomas melhoraram após um mês, com recuperação completa ao redor do quarto mês. Neste caso, a cintilografia de corpo inteiro com sestamibi- ${ }^{99 m}$ Tc foi decisiva na localização do tumor causador da osteomalácia oncogênica. (Arq Bras Endocrinol Metab 2008; 52/9:1505-1509)
\end{abstract}

Descritores: Tumor mesenquimal; Sestamibi--99mTc; Osteomalácia oncogênica; Hipofosfatemia; Medicina nuclear; Lipoma

\begin{abstract}
Oncogenic Osteomalacia: Localization of Underlying Peripheral Tumor with 99 ${ }^{\mathrm{m}}$ TC-Sestamibi Scintigraphy.

Oncogenic osteomalacia is a paraneoplastic syndrome usually induced by bone or soft tissue tumors. It is presented by the development of pain and fractures with hypophosphatemia, hyperphosphaturia, and inappropriate normal/low plasma $1,25(\mathrm{OH})_{2} \mathrm{D}_{3}$ concentration. After the removal of the tumor the complete resolution of all biochemical and clinical abnormalities is the main characteristic. A case of a 44-year-old female with difficulty in walking due to leg pain and generalized muscle weakness and hypophosphatemia, with relative hyperphosphaturia, is described. A whole-body ${ }^{99 m} \mathrm{Tc}$-sestamibi scintigraphy showed accumulation in the left thigh region, and a small tumor was detected by ultrasound examination. By removal of the tumor, a lipoma, the symptoms improved significantly after a month, with complete recovery by the fourth month. In this case, ${ }^{99 \mathrm{~m} T c-s e s t a m i b i}$ scintigraphy was useful in identifying the location of the tumor, which caused oncogenic osteomalacia. (Arq Bras Endocrinol Metab 2008; 52/9:1505-1509)
\end{abstract}

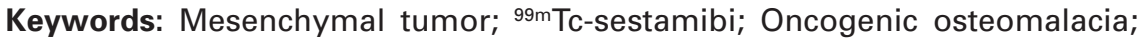
Hypophosphatemia; Nuclear medicine; Lipoma

\section{INTRODUÇÃO}

fraqueza muscular progressiva com comprometimento da marcha e acompanhada de quadro doloroso é geralmente investigada visando a apresentação de caso

\author{
Gleyne Lopes Kujew BiaginI \\ Priscilla Rizental Coutinho \\ THAISA HOFFMAN JONASSON \\ CRISTINA EMIKo UEDA \\ RICARDO RIBEIRO GAMA
}

Disciplina de Endocrinologia e Metabologia da Faculdade Evangélica do Paraná (Fepar) (GLKB); Serviço de Endocrinologia e Diabetes do Hospital Universitário Evangélico de Curitiba (PRC, THJ); Centro de Radioimunoensaio e Medicina Nuclear do Paraná (CEU); Serviço de Oncologia do Hospital Universitário Evangélico de Curitiba (RBG); Curitiba, PR, Brasil.

Recebido em 10/1/2008 Aceito em 17/9/2008 
exclusão de causas neurológicas. Um evento como a fratura de baixo impacto orienta para investigação ortopédica ou oncológica. Causas metabólicas, quando lembradas, são frequentemente relacionadas à osteoporose ou ao hiperparatireoidismo primário. As dosagens rotineiras de cálcio e fósforo séricos, na investigação destas moléstias, podem sinalizar para causa rara, porém curável, de osteomalácia oncogênica hipofosfatêmica $(\mathrm{OOH})$. Vários tumores benignos e malignos são descritos como causadores da $\mathrm{OOH}$. Os mais freqüentes são tumores variantes do tecido conjuntivo misto, os hemangiopericitomas, assim como simples hematomas organizados, hemangiomas ou mesmo nódulos fibrocísticos de mama (1). As células mesenquimais, que dão origem a estes elementos do tecido conjuntivo com potencial oncogênico e secretor de fatores fosfatúricos, as fosfatoninas, também se diferenciam em osteoblastos, miócitos, células de vasos linfáticos e sanguíneos, células do sistema nervoso periférico e adipócitos. A linhagem osteoblástica é a que possui evidência para a produção fisiológica de fosfatoninas (2). Os tumores de gordura, na sua maioria, assim como os de origem mesenquimal, são de baixo potencial metastático e inertes (3). O lipoma subcutâneo com esteatonecrose causando esta síndrome é inédito. A medicina nuclear tem, recentemente, sido apontada como a estratégia inicial ideal na localização destes tumores (4). A cintilografia óssea é útil, principalmente na pesquisa de lesões ósseas decorrentes do distúrbio metabólico, como fraturas de insuficiência, além da investigação de possíveis lesões ósseas causadoras dessa síndrome. Em casos de suspeita de lesões de partes moles causadoras de $\mathrm{OOH}$, está indicado o uso de outros radiofármacos, como o sestamibi- ${ }^{99 \mathrm{~m}} \mathrm{Tc}$, o cloreto de tálio-201, o octreotide- ${ }^{111}$ In e a tomografia por emissão de pósitrons (PET) com fluoro2-deoxiglicose marcada com flúor-18 (5-7).

\section{RELATO DO CASO}

A paciente SM, 44 anos, feminina, foi encaminhada em outubro de 2006 ao endocrinologista por apresentar dificuldade de locomoção, osteoporose na avaliação densitométrica e PTH elevado. O quadro teve início em 2003 com episódio de dor no dorso do pé esquerdo que a impossibilitou de deambular durante duas semanas. Na época foi submetida a tratamento com antiinflamatórios não-hormonais, obtendo relativa melhora. Seis meses após, já em 2004, apresentou dor difusa e notou fraqueza em membros inferiores com claudica- ção e dificuldade progressiva para a marcha. Procurou ortopedista, foi submetida a exames radiográficos com resultados compatíveis com a normalidade e manteve o uso de analgésicos e antiinflamatórios. Ao final de 12 meses estava dependente de muletas e impossibilitada de exercer suas funções domésticas. Por causa da fraqueza muscular foi encaminhada ao neurologista e ao oncologista. Após exaustiva investigação de imagem e bioquímica com resultados dentro da normalidade, exceto por discreta elevação da fosfatase alcalina, foi orientada a realizar biópsia óssea e a iniciar uso de derivados de morfina.

A lista de exames com resultados normais, alguns com várias repetições, a que foi submetida incluiu: hemograma, VHS, glicemia, transaminases, LDH, creatinina, função tireoidiana, parcial de urina, cálcio sérico urina de 24 horas, FAN, fator reumatóide, glicosúria, aminoacidúria, eletroforese de proteínas, proteína de Bence-Jones, beta-2 microglobulina, calcitonina, marcadores tumorais, biópsia de medula óssea, tomografias cervical, de tórax, abdome e pelve.

No início de 2006, a paciente sofreu fratura de colo de fềmur esquerdo, dando início a investigação para osteoporose. Concomitante a essa investigação foi solicitada cintilografia óssea com metilenodifosfonato marcado com tecnésio $99 \mathrm{~m}$ (MDP- ${ }^{99 \mathrm{~m} T c}$ ), que mostrou reação osteogênica no gradeado costal (especialmente os arcos posteriores), ulna esquerda, diáfise umeral direita e colo femoral direito. $\mathrm{O}$ achado de PTH elevado e posterior cintilografia cervical com sestamibi- ${ }^{99 \mathrm{~m}} \mathrm{Tc}$ negativa para tecido paratireoidiano hiperfuncionante foram os motivos do encaminhamento ao endocrinologista.

A história pregressa não revelava tendência a fraturas na infância, nem tampouco na família. A paciente julgava-se hígida até completar 40 anos. A fraqueza muscular era o sinal mais evidente ao exame físico. A paciente não conseguia, mesmo com ajuda, subir os degraus da escada de acesso à maca. A dor estava atenuada pelo uso, já frequente, de derivados de morfina. Além disso, notava-se obesidade centrípeta e pressão arterial levemente elevada ( 140 x $90 \mathrm{mmHg})$. O exame físico não evidenciou massas ou tumorações superficiais palpáveis.

Os valores do fósforo sérico de $1,4 \mathrm{mg} / \mathrm{dL}(2,6 \mathrm{a}$ $4,5)$ e urinário de $550 \mathrm{mg} / \mathrm{d}$ (400 a 1.200$)$ confirmaram a suspeita de $\mathrm{OOH}$. A taxa de reabsorção tubular de fósforo foi calculada em 71\% (normal acima de 85\%). A procura de imagem sugestiva de tumor mesenquimal 
na tomografia de face e de crânio foi negativa. $\mathrm{O}$ valor de $13,2 \mathrm{ng} / \mathrm{mL}$ da $25(\mathrm{OH}) \mathrm{VD}$ (desejável acima de 32 ) explicou o hiperparatireoidismo secundário e foi iniciada a reposição oral com calcidiol, calcitriol e fosfatos. A fosfatase alcalina mantinha-se elevada (347 UI/L). A ultra-sonografia de parede abdominal e de membros superiores, à procura de pequenas tumorações de subcutâneo, revelou imagem de hematoma no braço esquerdo, posteriormente identificado como local da aplicação intramuscular do antiinflamatório usado na semana anterior.

A cintilografia, desta vez solicitada com sestamibi${ }^{99 \mathrm{~m} T c}$ de corpo inteiro, dando ênfase à pesquisa de tumores mesenquimais, evidenciou a presença de pequena área focal de hipercaptação do radiotraçador no terço superior da coxa esquerda (Figura 1). A ultra-sonografia desta região demonstrou a presença de massa de 1,9 $\mathrm{cm}$ à profundidade de $2,5 \mathrm{~cm}$ sobre a superfície do músculo quadríceps, sendo sugestiva de esteatonecrose (Figura 2).

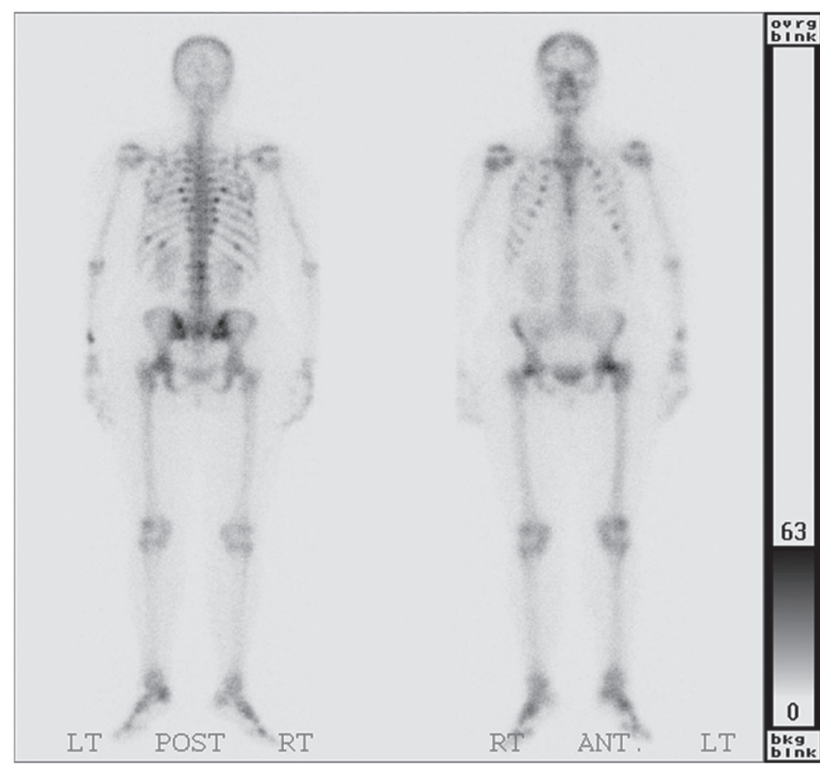

Figura 1. Cintilografia óssea de corpo inteiro com MDP99mTc demonstrando áreas de hiperconcentração do radiotraçador, com intensidade de captação variando de discreta a moderada, no gradeado costal bilateralmente, ulna esquerda, diáfise umeral direita, colo femoral direito e regiões metatarsianas, podendo corresponder a lesões ósseas decorrentes do distúrbio metabólico. A área de hipercaptação do radiofármaco no fêmur proximal esquerdo é compativel com aumento de remodelação óssea póstraumática/pós-manipulação cirúrgica.

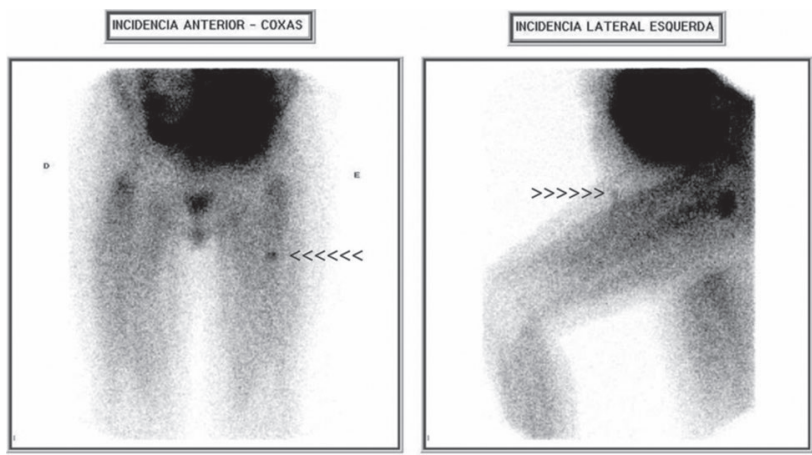

Figura 2. Cintilografia com sestamibi-99mTc. A imagem adquirida na projeção anterior demonstrou área focal de hiperconcentração do radiotraçador na coxa esquerda de difícil definição quanto à sua localização óssea ou em partes moles. A imagem adquirida na projeção lateral esquerda mostrou que a área de concentração anômala do radiofármaco era superficial, envolvendo somente as partes moles da região anterior do terço proximal da coxa esquerda.

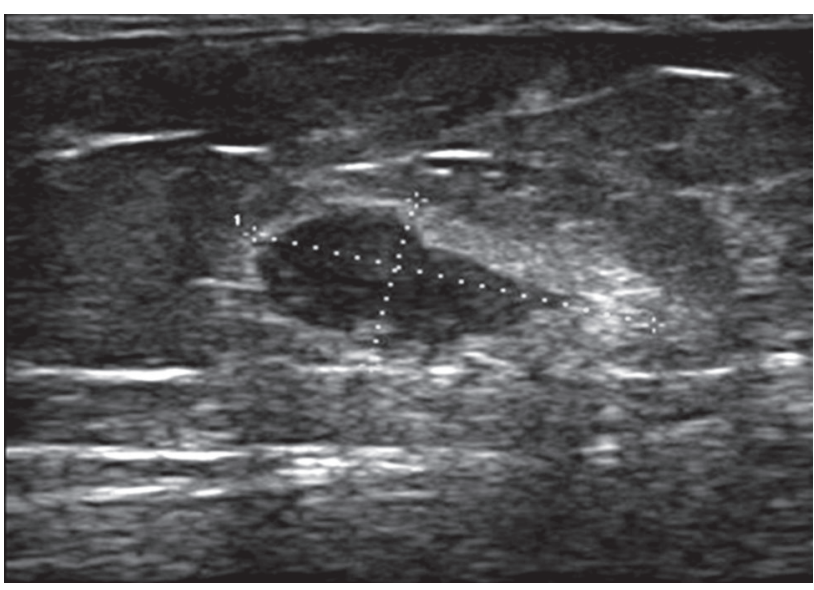

Figura 3. Ultra-sonografia superficial de coxa esquerda. Imagem em plano longitudinal demonstrando nódulo hipoecóico, de $2 \times 1,2 \mathrm{~cm}$, com margens ecogênicas, contornos bocelados e limites precisos, situado em plano subcutâneo, sugestiva de esteatonecrose.

Em dezembro de 2006, sob anestesia local, foi realizada a exérese do tumor. O diagnóstico foi confirmado pelo exame anatomopatológico como lipoma de 3,5 $\times 2,5 \times 2,0 \mathrm{~cm}$ com esteatonecrose, cuja microscopia mostrou adipócitos maduros e a presença de vacúolos de gordura de tamanhos variados, separados entre si por tecido conectivo fibroso, formando arranjo septado. Algumas células gordurosas anucleadas circundadas por células gigantes histiocíticas e agregados de histiócitos e fagócitos espumosos caracterizaram a esteatone- 
crose. O painel de imunoistoquímica obtido revelou ausência de células reatoras para sinaptofisina e cromogranina A.

Aguardava-se a melhora rápida dos sintomas, em torno da primeira semana, como relatada na literatura, caso fosse o tumor ressecado o secretor de fosfatoninas. No entanto, houve piora do quadro geral doloroso. As dores ósseas anteriormente mais graves em membros inferiores reduziram, porém deram lugar para queixas relacionadas ao esqueleto axial, na porção anterior de tórax e na coluna toracolombar. O resultado do fósforo sérico, entretanto, coletado na segunda semana, mostrou-se normal $(4,5 \mathrm{mg} / \mathrm{dL})$. Progressivamente, após a quarta semana, a paciente notou melhora da força muscular, iniciando deambulação sem necessidade de apoio. No segundo mês do pós-operatório, a paciente já estava exercendo suas funções domésticas habituais com desenvoltura, e, após o terceiro mês, já pôde comparecer à consulta clínica sem acompanhante. Ao final do quarto mês, tendo retornado a todas as atividades habituais prévias à doença; diminuiu $12 \mathrm{~kg}$, perdendo o aspecto da obesidade centrípeta.

\section{DISCUSSÃo}

A hipofosfatemia pode ser causada pela má absorção, vista em algumas deficiências vitamínicas e doenças intestinais, além do uso de antiácidos quelantes de fósforo. Tanto a má absorção quanto a redistribuição do íon em situações agudas, como a síndrome da realimentação e alcalose respiratória, cursam com hipofosfatemia, porém a excreção de fósforo está também diminuída. As causas crônicas que envolvem o aumento da perda renal de fósforo incluem o uso de diuréticos do tipo inibidores da anidrase carbônica, o hiperparatireoidismo, a resistência à ação da vitamina $\mathrm{D}$ e as desordens genéticas congênitas ou adquiridas (8). A OOH é uma síndrome adquirida, caracterizada pela presença de hipofosfatemia, hiperfosfatúria e causada por tumor de tecido conjuntivo misto ou ósseo, que secreta fatores reguladores do transporte do fósforo e de sua homeostase, as fosfatoninas (1).

O metabolismo do fósforo é regulado pelo conjunto de hormônios. O paratormônio (PTH) e a $1,25(\mathrm{OH})_{2} \mathrm{D}_{3}$ são os mais bem estudados. As fosfatoninas ou fosfoproteínas regulam não só o metabolismo do fósforo como também o do calcitriol $(2,9)$. Por meio da inibição da enzima 1- $\alpha$-hidroxilase, promovem menor conversão renal de $25(\mathrm{OH})_{2} \mathrm{VD}$ em $1,25(\mathrm{OH})_{2} \mathrm{D}_{3}(10,11)$. Esta ação determina queda nos níveis de calcitriol a despeito de baixos níveis de fósforo. Independente do PTH também inibem a reabsorção de fosfato, por meio da inativação do co-transportador sódio-fósforo (NaPT-2) no túbulo renal, com conseqüente hiperfosfatúria e espoliação de seus níveis plasmáticos $(9,10)$.

O fator 23 de crescimento do fibroblasto (FGF$23)$ é a fosfatonina responsável pela depleção do fosfato na maior parte dos casos relatados $(11,12)$. A partir do melhor entendimento do papel da FGF-23, outras fosfatoninas, também responsáveis pelo distúrbio hipofosfatêmico, foram descritas. A proteína Frizzled relacionada (FRP4), que faz parte do complexo receptor das proteínas Wnt, a MEPE (matriz extracelular de fosfoglicoproteína), assim como o FGF-7 (fator 7 de crescimento do fibroblasto), já foram identificados como expressos de maneira exagerada em diversas desordens hipofosfatêmicas (13).

A $\mathrm{OOH}$, um subtipo de osteomalácia adquirida, é caracterizada como fenômeno paraneoplásico, no qual a doença musculoesquelética tem maior significância clínica que o tumor propriamente dito, sendo totalmente revertida com a retirada completa deste tumor. Estes, em geral, são benignos, pequenos, de origem mesenquimal e raramente detectados em exames radiográficos de rotina (8). Existe, de maneira anedótica, relato de resolução do caso após a retirada de simples hematoma (14).

A apresentação clínica é idêntica à da osteomalácia hipofosfatêmica ligada ao X e à osteomalácia autossômica dominante, com os achados clínicos típicos de diminuição da mineralização óssea, dores ósseas, fraturas e osteopenia, porém em idade mais avançada. As queixas de fraqueza muscular, na $\mathrm{OOH}$, são mais intensas, assim como a presença de fratura e a dor óssea são mais freqüentes e graves (15-17).

A investigação diagnóstica deve incluir exames de imagem, inicialmente dirigida para a região de cabeça e pescoço, onde os tumores se localizam de maneira mais predominante. A recomendação feita, até recentemente, era a de realizar tomografia computadorizada e ressonância magnética de mandíbula e seios da face, não obstante a baixa sensibilidade de ambos os métodos em localizar tais neoplasias, principalmente as do tecido conjuntivo $(5-7,18)$.

Vários métodos cintilográficos têm sido descritos como sensíveis para localização de pequenos tumores. 
Tanto a cintilografia óssea, após injeção de hidroximetileno-difosfonato marcado com tecnésio- ${ }^{-99 \mathrm{~m}}$ quanto a cintilografia com octreotideo ou pentetreotideo marcados com indio ${ }^{111}$ são opções diagnósticas $(17,18)$.

A escolha pelo sestamibi- ${ }^{-99 \mathrm{~m}} \mathrm{Tc}$, nesta paciente, foi fundamentada nas vantagens apresentadas sobre os demais traçadores descritos na investigação de tumores mesenquimais causadores de $\mathrm{OOH}$. Expõe menor dose de radiação ao paciente e maior disponibilidade em nosso meio. Com sucesso, também, na localização destes pequenos tumores, mostra tendência de que este método venha a ser o mais adequado para a pesquisa inicial $(4,5)$.

O curso da doença é marcado pela reversão completa do quadro metabólico e osteomuscular com a retirada do tumor. O quadro bioquímico se altera em horas ou em poucos dias. Quando disponível, a dosagem sérica dos níveis de FGF-23 serve como marcador de cura, uma vez que, na maioria dos pacientes com remissão pós-cirúrgica, existe também redução concomitante nos níveis desta fosfatonina (17). Neste caso não foi possível a dosagem de FGF-23.

Atualmente, o quadro clínico e bioquímico da paciente confirmam a cura da $\mathrm{OOH}$, após a retirada do lipoma de coxa esquerda há 18 meses.

Ao autores declaram não haver conflitos de interesse científico neste artigo.

\section{REFERÊNCIAS}

1. Rauch F, Scheinman SJ, Agus ZS, Drezner MK. Hereditary hypophosphatemic rickets and tumor-induced osteomalacia. UpTo Date ${ }^{\circledR}$ [acessado em 2008 Jan 15]. Disponível em: $<\mathrm{http} / /$ :www.uptodate.com>.

2. Berndt TJ, Schiavi S, Kumar R. Phosphatonins and the regulation of phosphorus homeostasis. Am J Physiol Renal Physiol. 2005;289:F1170-82.

3. Weiss SW. Lipomatous tumors. Monogr Pathol. 1996;38:207-39

4. Hodgson SF, Clarke BL, Tebben PJ, Mullan BP, Cooney WP, Shives TC. Oncogenic osteomalacia: localization of underlying peripheral mesenchymal tumors with use of Tc $99 \mathrm{~m}$ sestamibi scintigraphy. Endocr Pract. 2006;12(1):35-42.
5. Kimizuka T, Ozaki Y, Sumi Y. Usefulness of 201TI and 99mTc MIBI scintigraphy in a case of oncogenic osteomalacia. Ann Nucl Med. 2004;18(1):63-7.

6. Seufert J, Ebert K, Müller J, et al. Octreotide therapy for tumorinduced osteomalacia. N Engl J Med. 2001;345(26):1883-8.

7. Hesse E, Moessinger E, Rosenthal H, Laenger F, Brabant G, Petrich T. Oncogenic osteomalacia: exact tumor localization by co-registration of positron emission and computed tomography. J Bone Miner Res. 2007;22(1):158-62.

8. Agus ZS, Goldfarb S, Sheridan AM. Causes of hypophosphatemia. UpTo Date ${ }^{\circledR}$ [acessado em 2008 Jan 18]. Disponível em: $<$ http//:www.uptodate.com>.

9. Henry M. Kronenberg. NPT2a - The key to phosphate homeostasis. N Engl J Med. 2002;347:1022-4.

10. Gupta A, Winer K, Econs MJ, Marx SJ, Collins MT. FGF-23 is elevated by chronic hyperphosphatemia. J Clin Endocrinol Metabol. 2004;89(9):4489-92.

11. Sommer S, Berndt T, et al. The phosphatonins and the regulation of phosphate transport and vitamin D metabolism. J Steroid Biochem Mol Biol. 2007;103(3-5):497-503.

12. Jonsson $K B$, Zahradnik $R$, Larsson T. Fibroblast growth factor 23 in oncogenic osteomalacia and X-linked hypophosphatemia. N Engl J Med. 2003;1656-63.

13. White EK, Larsson TE, Econs MJ. The roles of specific genes implicated as circulating factors involved in normal and disordered phosphate homeostasis: Frizzled related protein-4, matrix extracellular phosphoglycoprotein and fibroblast growth factor 23. Endocr Rev. 2006;27(3):221-41.

14. Colt E, Gopan T, Chong HS. Oncogenic osteomalacia cured by removal of an organized hematoma. Endocr Pract. 2005;11 (3):190-3.

15. Mechica JB. Raquitismo e osteomalacia. Arq Bras Endocrinol Metab. 1999;43(6),457-66.

16. Moreira RO, Leal CTS, Lacativa PGS, Figueiredo JG, Lima MB, Farias ML. Hiperparatireoidismo associado a osteomalácia hipofosfatêmica do adulto: relato de caso e revisão da literatura. Arq Bras Endocrinol Metab. 2006;50(1):150-5.

17. Carpenter TO. Oncogenic osteomalacia - a complex dance of factors. N Engl J Med. 2003;348(17):1705-8.

18. Imanishi $Y$, Nakatsuka K, Nakayama T, Okamura T, Kobayashi $\mathrm{K}$, Nakayama K, et al. False-positive magnetic resonance imaging skeletal survey in a patient with sporadic hypophosphatemic osteomalacia. J Bone Miner Metab. 2003;21:57-9.

\section{Endereço para correspondência:}

Gleyne Lopes Kujew Biagini

Rua da Paz, 195, cj. 220

80060-160 Curitiba PR

Email: endocrino1999@hotmail.com 